\title{
OPEN Circulating tumour cells at baseline and late phase of treatment provide prognostic value in breast cancer
}

\author{
Shuyun Pang ${ }^{1,5}$, Hanjun $\mathrm{Li}^{2,5}$, Shu $\mathrm{Xu}^{1,4,5}$, Liying Feng ${ }^{1}$, Xueping $\mathrm{Ma}^{1}$, Yanan $\mathrm{Chu}^{1}$, \\ Bingjie Zou ${ }^{1,3 凶}$, Shaohua Wang ${ }^{2 \square}$ \& Guohua Zhou ${ }^{1,3 凶}$
}

To determine the prognostic value of the timing of circulating breast tumour cell measurement during treatment, peripheral blood from 164 patients with breast disease was collected. Circulating tumour cells (CTCs) were enriched by using immunomagnetic nanospheres (IMNs) and were identified by using immunofluorescent staining. The CTC shows nuclear-positive, EpCAM-positive, CK19-positive, and CD45-negative. Patients with CTC positivity (>19/7.5 mL blood) had shorter progression-free survival (PFS) and overall survival (OS) than those with negative results ( $\leq 19 / 7.5 \mathrm{~mL}$ blood) at baseline. Surgery caused an increase in the number and prevalence of CTCs, and the effect disappeared on day 14 after surgery. During adjuvant chemotherapy, CTCs detected before therapy was only correlated with PFS; however, CTCs at the end of adjuvant chemotherapy were correlated with both PFS and OS. The PFS and OS of the CTC-positive group were significantly shorter than those of the CTC-negative group at the end-point follow-up visit. The prognostic value of CTCs at different measurement time points was demonstrated during breast cancer treatment. Surgery and chemotherapy affected the prevalence of CTCs, leading to different prognostic relevance of CTCs at different treatment stages. CTCs detected at baseline or in the late phase of treatment are preferable for prognosis.

Circulating tumour cells (CTCs) are released from tumours into the vascular system. CTCs show characteristics of tumour and stem cell capability ${ }^{1,2}$, which is related to metastasis. Increasing evidence has shown that CTCs are the seeds of tumour metastasis ${ }^{2-5}$. Therefore, CTCs are potential biomarkers for diagnosis ${ }^{6}$ and prognosis ${ }^{7,8}$ of tumours. In addition, CTC detection is non-invasive, enabling monitoring of tumour progression ${ }^{9}$ and timely evaluation of efficacy ${ }^{3}$.

CTCs as biomarkers were first used in the prognosis of metastatic breast cancer ${ }^{7}$. Many prospective studies including large-scale multi-centre research confirmed that the number of CTCs over 5/7.5 ml blood detected by CellSearch system in metastatic breast cancer was associated with poor prognosis ${ }^{7,10-12}$. Moreover, CTCs have been incorporated into the 7th edition of the American Joint Committee on Cancer staging system for breast cancer as an index of distant metastases ${ }^{13}$. The number of CTCs detected by CellSearch system over 1/7.5 ml blood was also confirmed to be prognostic for progression-free survival (PFS) and overall survival (OS) in patients with non-metastatic breast cancer ${ }^{14}$.

However, many studies on the prognostic relevance of CTCs in breast cancer have used the number of CTCs at one time point before or after a certain therapy such as surgery ${ }^{15}$ and chemotherapy ${ }^{16}$ for correlation analysis. In fact, the number of CTCs varies during treatment, and the therapy could affect the number of CTCs. For example, the number of CTCs increased during the following 3-4 days after surgery ${ }^{17}$. One study showed that PFS and OS were significantly associated with the number of CTCs after adjuvant chemotherapy but not at 1-week post-surgery ${ }^{18}$, indicating that the measurement time point could affect the prognostic value of CTCs. Therefore, it is necessary to study the prognostic value of the timing of CTC detection during the treatment of

\footnotetext{
${ }^{1}$ Department of Clinical Pharmacy, State Key Laboratory of Analytical Chemistry for Life Science and Jiangsu Key Laboratory of Molecular Medicine, Jinling Hospital, Medical School of Nanjing University, Nanjing 210002, China. 'Department of General Surgery, Jinling Hospital, Medical School of Nanjing University, Nanjing 210002, China. ${ }^{3}$ School of Pharmaceutical Science, Southern Medical University, Guangzhou 510515, China. ${ }^{4}$ School of Basic Medical Science and Clinical Pharmacy, China Pharmaceutical University, Nanjing 210000, China. ${ }^{5}$ These authors contributed equally: Shuyun Pang, Hanjun Li and Shu Xu. ${ }^{\boxplus}$ email: zbj523@163.com; wanglaifu2@126.com; ghzhou@nju.edu.cn
} 
Bright field

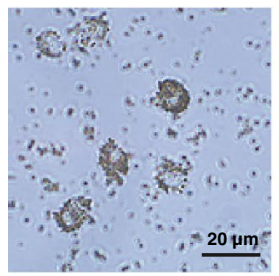

Nucleus-DAPI

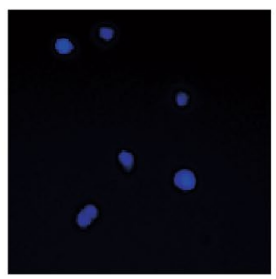

CK19-FITC

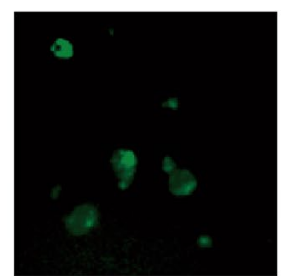

CD45-APC

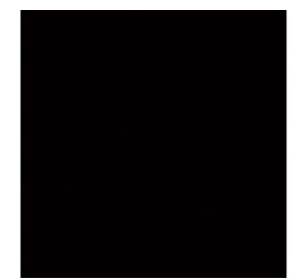

Merge

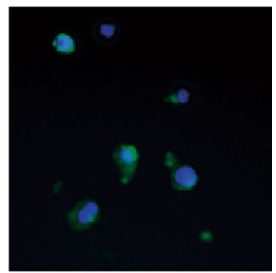

Figure 1. Images of IMN-captured and immunofluorescence-stained CTCs from a patient peripheral blood specimen.

breast cancer. Although some studies have compared the prognostic relevance of CTCs in breast cancer before and after treatment ${ }^{8,19,20}$, few studies have included different treatment stages.

On the other hand, the capture efficiency of the CTC enrichment method also affects its prognostic accuracy. Although epitope-based CTC enrichment methods (such as CellSearch system) may miss some CTCs that do not express the corresponding antigens, they are still the most commonly used CTC enrichment methods because of the simple and convenient operation. Therefore, when using epitope-based CTC enrichment method to study the prognostic value of CTC, it is necessary to choose a CTC enrichment method with high capture efficiency, so as to avoid further loss of CTC. The capture efficiency of epitope-based CTC enrichment method is mainly affected by the surface to volume ratio of the capture medium. Magnetic nanoparticles have a relatively high surface to volume ratio, inducing the high binding ability and CTC capture efficiency. However, the magnetic response of magnetic nanoparticles is not fast enough, and they suffer higher loss rate. Immunomagnetic nanospheres (IMNs) with quick magnetic response overcome this defect of magnetic nanoparticles ${ }^{21}$. IMNs are made of PstAAm-COOH as the core, and coated with 5 layers of nano- $\gamma-\mathrm{Fe}_{2} \mathrm{O}_{3}$ to get a large magnetic saturation value ${ }^{21}$, so that the IMNs could be captured within 1 min with commercial magnetic medium ${ }^{21}$. Therefore, the whole progress of CTC enrichment could be completed within 5 min with $\sim 94 \%$ recovery rate ${ }^{21}$.

Therefore, by using IMNs to capture the CTC, the prognostic relevance of CTCs at the time point of presurgery, pre-adjuvant chemotherapy, final adjuvant chemotherapy, and perioperative period was demonstrated to clarify which time points the CTC has prognostic value.

\section{Results}

Optimization of CTC enrichment method and threshold demarcation. Quick-response IMNs can capture tumour cells in blood with an efficiency of more than $94 \%$ after only 5 min of incubation ${ }^{21}$. Therefore, it is a rapid and sensitive CTC detection method. However, the previously described method recommends using $1.5 \mathrm{~mL}$ of blood to detect CTCs, which is less than the sample volume $(7.5 \mathrm{~mL})$ in the CellSearch system. Because a larger volume of blood could increase the sensitivity of CTC detection, we increased the blood sample volume to $7.5 \mathrm{~mL}$ for the IMN-based CTC detection.

To accurately and efficiently enrich CTCs in a $7.5 \mathrm{~mL}$ blood sample, we optimised the capture conditions by performing experiments with different concentrations of IMNs and incubation times, and then compared the capture recovery rate of each condition. The recovery rate was calculated by dividing the number of captured MCF-7 cells by the number of input MCF-7 cells. As shown in Fig. S1a, $0.13 \mathrm{mg} / \mathrm{mL}$ IMNs gave a $97.1 \%$ recovery rate, which was higher than that of $0.07 \mathrm{mg} / \mathrm{mL}$ IMNs $(85.7 \%$ recovery rate) and $0.20 \mathrm{mg} / \mathrm{mL} \mathrm{IMNs}(91.4 \%$ recovery rate). Incubation times of $5 \mathrm{~min}$ and $10 \mathrm{~min}$ showed recovery rates of over 95\% (Fig. S1b), but $1 \mathrm{~min}$ and $15 \mathrm{~min}$ incubation times showed lower recovery rates. Therefore, $0.13 \mathrm{mg} / \mathrm{mL}$ IMNs and 5 -min incubation were used in subsequent experiments.

To investigate the capture specificity of IMNs, three kinds of epithelial tumour cells (HCT116, A549, and MCF-7) and leukocytes were added to PBS $(7.5 \mathrm{~mL})$ and captured by IMNs. The recovery rates of all epithelial tumour cells were over $92 \%$, but the recovery rate of leukocytes was $16.6 \%$ (Fig. S1c). The non-specific capture of leukocytes may be caused by the non-specific absorption of IMNs. Therefore, we further increased the washing time, and non-specific adsorption was reduced by washing multiple times (Fig. S1d). In addition, leukocytes could be distinguished by CD45 staining, thus, it did not affect CTC counts. Leukocyte residues are inevitable. But it is no significant difference in the number of leukocytes between the breast cancer and healthy donors (Fig. S1e).

Anti-EpCAM antibody also captures epithelial non-tumour cells in the blood. Thus cells displaying nuclearpositive, CK19-positive, and CD45-negative by immunocytochemistry, were also observed in the blood samples from healthy donors (Fig. S1f). Blood samples from 19 healthy donors were collected and tested for CTC-positive threshold demarcation, and the results showed that the mean epithelial cell count was 8.89, and the standard deviation (SD) was 3.72. Therefore, the threshold of the CTC-positive sample was 19, calculated by adding the mean epithelial cell counts and three times the SD.

CTCs were detected in the peripheral blood of the patients. CTCs were wrapped in magnetic beads and were nuclear-positive, EpCAM-positive, CK19-positive, and CD45-negative (Figs. 1 and S3). Interestingly, we found that CTCs were also detected in $42.3 \%$ of the patients who were diagnosed with benign breast diseases (Figure S1f). This might be related to the presence of epithelial cells in the blood of different patients due to personal condition or medication, indicating that CTCs are not a good biomarker for diagnosing benign breast diseases. 
Patient characteristics and CTC prevalence at baseline. Between 2015 and 2016, 138 patients with breast cancer were enrolled in the study. CTC detection was successfully performed in 110 patients $(79.7 \%)$ before a new line of treatment, except for 28 patients (20.3\%), whose blood samples were not qualified or the information was not collected (Fig. S2). The CTCs enumeration results are summarized in Table S1. The characteristics of patients with CTC detection results at baseline are summarised in Table 1 . Of the 110 patients, 55 (50.0\%) were CTC-negative ( $\leq 19 / 7.5 \mathrm{~mL}$ blood) and 55 (50.0\%) were CTC-positive (>19/7.5 mL blood). The age range of patients was $23-84$ years, and the average age of the patients was 52.7 . Eleven patients (10.0\%) had ductal carcinoma in situ, 88 patients $(80.0 \%)$ had invasive ductal carcinoma, and 11 patients (10.0\%) had other pathological types of breast cancer, including neuroendocrine carcinoma, invasive lobular carcinoma, metaplastic carcinoma, and mixed carcinoma. CTCs were associated with distant metastasis $(P=0.0429)$. Patients with distant metastasis had a higher prevalence of CTCs, which is in line with the status quo. Most metastatic breast cancers have a higher CTC prevalence than non-metastatic breast cancers in different studies ${ }^{22-25}$. There were no significant differences in intrinsic subtype, stage, risk categories, tumour size, lymph node status, oestrogen receptor (ER) status, progesterone receptor (PR) status, human epidermal growth factor receptor 2 (HER2) status, and Ki67 status between the CTC-positive and CTC-negative groups of patients $(P>0.05)$.

Prognostic relevance of CTC level at baseline. Survival data were obtained for the 110 patients available for evaluation. The median follow-up duration was 45 months. A total of 37 patients (33.6\%) developed disease recurrence, and 20 (18.2\%) died during follow-up. Fifty-five patients (50.0\%) were CTC-positive compared with 55 patients $(50.0 \%)$ were CTC-negative. Patients with CTC positivity had a significantly shorter time to progression than the patients with CTC negativity $(P=0.0002$; hazard ratio $[\mathrm{HR}], 3.56 ; 95 \%$ confidence interval [CI], 1.86-6.82), and patients with CTC positivity had a significantly shorter time to death than patients with CTC negativity ( $P=0.0011$; HR, 4.98; 95\% CI, 2.06-12.02) (Fig. 2a and b). These results indicated that the presence of CTCs at baseline was associated with an unfavourable prognosis.

Various clinicopathological prognostic factors, including baseline CTCs, intrinsic subtype, histology, stage, risk categories, tumour size, lymph node status, visceral metastases, ER status, PR status, HER2 status, and Ki67 status were considered for the univariate Cox regression analysis (Table 2). Baseline CTCs $(P=0.000 ; \mathrm{HR}, 3.93$; 95\% CI, 1.84-8.39), stage ( $P=0.000 ; \mathrm{HR}, 2.17 ; 95 \% \mathrm{CI}, 1.57-3.00)$, and risk categories $(P=0.000 ; \mathrm{HR}, 3.68$; 95\% CI 1.94-7.00) were significantly correlated. CTC positivity at baseline, high stage, and high-risk categories signified a shorter PFS. In Cox multivariate analysis, baseline CTCs ( $P=0.002$; HR, 3.63; 95\% CI, 1.60-8.27) and stage $(P=0.031$; HR $1.75 ; 95 \% \mathrm{CI}, 1.05-2.90)$ were significantly correlated (Table 2$)$, indicating that both baseline CTCs and stage were independent factors for PFS. For OS, baseline CTCs $(P=0.003$; HR, 6.50; $95 \%$ CI, 1.89-22.36), stage ( $P=0.000 ; \mathrm{HR}, 4.22 ; 95 \% \mathrm{CI}, 2.32-7.68)$, risk categories $(P=0.000 ; \mathrm{HR}, 8.99 ; 95 \% \mathrm{CI}$ 3.04-26.57), and ER status ( $P=0.049$; HR, 0.41; 95\% CI, 0.17-1.00) were related to poor prognosis. Similarly, baseline CTCs $(P=0.042$; HR, 4.35; 95\% CI, 1.05-17.98) and stage $(P=0.003$; HR, 3.79; 95\% CI, $1.57-9.14)$ were independent factors for OS in multivariate analysis.

CTCs in the perioperative period and their prognostic relevance. Surgery can affect the number of CTCs in a patient's blood ${ }^{17}$. We investigated the CTCs during the perioperative period. The number of CTCs significantly increased on the first day after surgery $(P=0.0001)$ (Fig. S4a), and the CTC prevalence increased from $44.6 \%$ to $65.8 \%(P=0.0073)$. The prevalence increase persisted until $7 \mathrm{~d}$ after surgery $(P=0.0147)$, and the number of CTCs levelled $(P=0.0634)$ (Fig. S4b). There was no significant difference between the number of CTCs $14 \mathrm{~d}$ after surgery and the number of CTCs before surgery $(P=0.3718)$, and the CTC prevalence decreased to $54.2 \%$ at $14 \mathrm{~d}$ after surgery $(P=0.2899)$ (Fig. S4c). Therefore, CTC count varied in the perioperative period. It is necessary to further investigate the prognostic value of CTCs at different measurement time points during the perioperative period.

As Fig. 3 shows, the PFS $(P=0.0036 ; \mathrm{HR}, 4.05 ; 95 \% \mathrm{CI}, 1.62-10.10)$ and $\mathrm{OS}(P=0.0020 ; \mathrm{HR}, 10.71 ; 95 \%$ CI, 2.39-48.06) of preoperatively CTC-positive patients were significantly shorter than those of CTC-negative patients. However, there was no significant difference in PFS $\left(P_{1 \mathrm{~d}}=0.9868 ; \mathrm{HR}_{1 \mathrm{~d}}, 0.99 ; 95 \% \mathrm{CI}_{1 \mathrm{~d}}, 0.34-2.90\right.$; $\left.P_{7 \mathrm{~d}}=0.6145 ; \mathrm{HR}_{7 \mathrm{~d}}, 1.50 ; 95 \% \mathrm{CI}_{7 \mathrm{~d}}, 0.35-6.53\right)$ and $\mathrm{OS}\left(P_{1 \mathrm{~d}}=0.9883 ; \mathrm{HR}_{1 \mathrm{~d}}, 0.99 ; 95 \% \mathrm{CI}_{1 \mathrm{~d}}, 0.18-5.41\right.$; $\left.P_{7 \mathrm{~d}}=0.9560 ; \mathrm{HR}_{7 \mathrm{~d}}, 0.93 ; 95 \% \mathrm{CI}_{7 \mathrm{~d}}, 0.08-10.61\right)$ between CTC-positive and CTC-negative patients on days 1 and 7 after surgery. However, CTCs detected $14 \mathrm{~d}$ after surgery were related to PFS ( $P=0.0412 ; \mathrm{HR}, 2.74 ; 95 \% \mathrm{CI}$, $0.88-8.55)$, indicating that the variation of CTC counts caused by surgery could affect the prognostic relevance of CTCs.

Prognostic relevance of CTCs before initial and final adjuvant chemotherapy. After surgery, many patients received adjuvant chemotherapy. CTCs in the blood samples from 60 patients were measured before initial adjuvant chemotherapy and before the final adjuvant chemotherapy to investigate the effect of adjuvant chemotherapy on the prognostic relevance of CTCs. The prevalence of CTCs before adjuvant chemotherapy (51.7\%) was higher than that after adjuvant chemotherapy (46.7\%), indicating that chemotherapy had an impact on CTCs. The PFS of CTC-positive patients before the first chemotherapy was significantly shorter than that of CTC-negative patients $(P=0.0314 ; \mathrm{HR}, 2.92 ; 95 \% \mathrm{CI}, 1.16-7.37)$ (Fig. 4a), but there was no significant difference in OS ( $P=0.0930$; HR, 3.51; 95\% CI, 0.95-12.97) (Fig. 4b). After adjuvant chemotherapy, CTC-positive patients had significantly shorter PFS $(P=0.0066$; HR, 3.48; 95\% CI, 1.47-9.59) and OS $(P=0.0062$; HR 10.27; 95\% CI, 2.75-38.31) than CTC-negative patients (Fig. $4 \mathrm{c}$ and d), indicating that CTCs after adjuvant chemotherapy are more associated with prognosis. Therefore, CTCs in the late phase of disease treatment have prognostic value. 


\begin{tabular}{|c|c|c|c|c|}
\hline & All patients $(\mathrm{N}=110)$ & Baseline CTC negative $(\mathrm{N}=55)$ & Baseline CTC positive $(\mathrm{N}=55)$ & $P$ value \\
\hline Age & $52.7(23-84)$ & $53.6(34-84)$ & $51.7(23-79)$ & $0.3545^{\mathrm{a}}$ \\
\hline Histology & & & & $0.1482^{c}$ \\
\hline Ductal carcinoma in situ & 11 & $3(27.2 \%)$ & $8(72.8 \%)$ & \\
\hline Invasive ductal carcinoma & 88 & $48(54.5 \%)$ & $40(45.5 \%)$ & \\
\hline Other types & 11 & $4(36.4 \%)$ & $7(63.6 \%)$ & \\
\hline Intrinsic subtype & & & & $0.4874^{\mathrm{c}}$ \\
\hline Luminal A-like & 25 & $11(44.0 \%)$ & $14(56.0 \%)$ & \\
\hline Luminal B-like (HER2 negative) & 32 & $13(40.6 \%)$ & $19(59.4 \%)$ & \\
\hline Luminal B-like (HER2 positive) & 24 & $15(62.5 \%)$ & $9(37.5 \%)$ & \\
\hline Erb-B2 overexpression & 19 & $10(52.6 \%)$ & $9(47.4 \%)$ & \\
\hline Basal-like & 10 & $6(60.0 \%)$ & $4(40.0 \%)$ & \\
\hline Stage & & & & $0.2702^{\mathrm{b}}$ \\
\hline 0 & 8 & $2(25.0 \%)$ & $6(75.0 \%)$ & \\
\hline I & 19 & $11(57.9 \%)$ & $8(42.1 \%)$ & \\
\hline II & 38 & $25(65.8 \%)$ & $13(34.2 \%)$ & \\
\hline III & 18 & $8(44.4 \%)$ & $10(55.6 \%)$ & \\
\hline IV & 20 & $6(30.0 \%)$ & $14(70.0 \%)$ & \\
\hline N/A & 7 & & & \\
\hline Risk categories & & & & $0.4010^{\mathrm{b}}$ \\
\hline Low risk & 8 & $2(25.0 \%)$ & $6(75.0 \%)$ & \\
\hline Intermediate risk & 62 & $39(62.9 \%)$ & $23(37.1 \%)$ & \\
\hline High risk & 34 & $13(38.2 \%)$ & $21(61.8 \%)$ & \\
\hline N/A & 6 & & & \\
\hline Tumour (TNM staging) & & & & $0.5570^{\mathrm{b}}$ \\
\hline 0 & 7 & $2(28.6 \%)$ & $5(71.4 \%)$ & \\
\hline 1 & 37 & $19(51.4 \%)$ & $18(48.6 \%)$ & \\
\hline 2 & 55 & $31(56.4 \%)$ & $24(43.6 \%)$ & \\
\hline 3 & 7 & $1(14.3 \%)$ & $6(85.7 \%)$ & \\
\hline 4 & 2 & $2(100 \%)$ & $0(0 \%)$ & \\
\hline N/A & 2 & & & \\
\hline Node (TNM staging) & & & & $0.1483^{\mathrm{b}}$ \\
\hline 0 & 53 & $29(54.7 \%)$ & $24(45.3 \%)$ & \\
\hline 1 & 16 & $11(68.7 \%)$ & $5(31.3 \%)$ & \\
\hline 2 & 15 & $6(40.0 \%)$ & $9(60.0 \%)$ & \\
\hline 3 & 12 & $4(33.3 \%)$ & $8(66.7 \%)$ & \\
\hline $\mathrm{N} / \mathrm{A}$ & 14 & & & \\
\hline Metastasis (TNM staging) & & & & $0.0429^{c}$ \\
\hline 0 & 89 & $49(55.1 \%)$ & $40(44.9 \%)$ & \\
\hline 1 & 20 & $6(30.0 \%)$ & $14(70.0 \%)$ & \\
\hline $\mathrm{N} / \mathrm{A}$ & 1 & & & \\
\hline ER & & & & $0.8352^{\mathrm{c}}$ \\
\hline Negative & 33 & $17(51.5 \%)$ & $16(48.5 \%)$ & \\
\hline Positive & 77 & $38(49.4 \%)$ & $39(50.6 \%)$ & \\
\hline PR & & & & $0.8472^{c}$ \\
\hline Negative & 63 & $31(49.2 \%)$ & $32(50.8 \%)$ & \\
\hline Positive & 47 & $24(51.1 \%)$ & $23(48.9 \%)$ & \\
\hline Her-2 & & & & $0.3286^{c}$ \\
\hline Negative & 67 & $31(46.3 \%)$ & 36 (53.7\%) & \\
\hline Positive & 43 & $24(55.8 \%)$ & $19(44.2 \%)$ & \\
\hline Ki67 & & & & $0.5541^{\mathrm{c}}$ \\
\hline$\leq 20 \%$ & 41 & $19(46.3 \%)$ & $22(53.7 \%)$ & \\
\hline$>20 \%$ & 69 & $36(52.2 \%)$ & $33(47.8 \%)$ & \\
\hline
\end{tabular}

Table 1. Patient characteristics and prevalence of CTCs. ${ }^{a} P$ value from Mann-Whitney test. ${ }^{b} P$ value from Pearson's chi-squared test for trend. ${ }^{c} P$ value from Pearson's chi-squared test. 

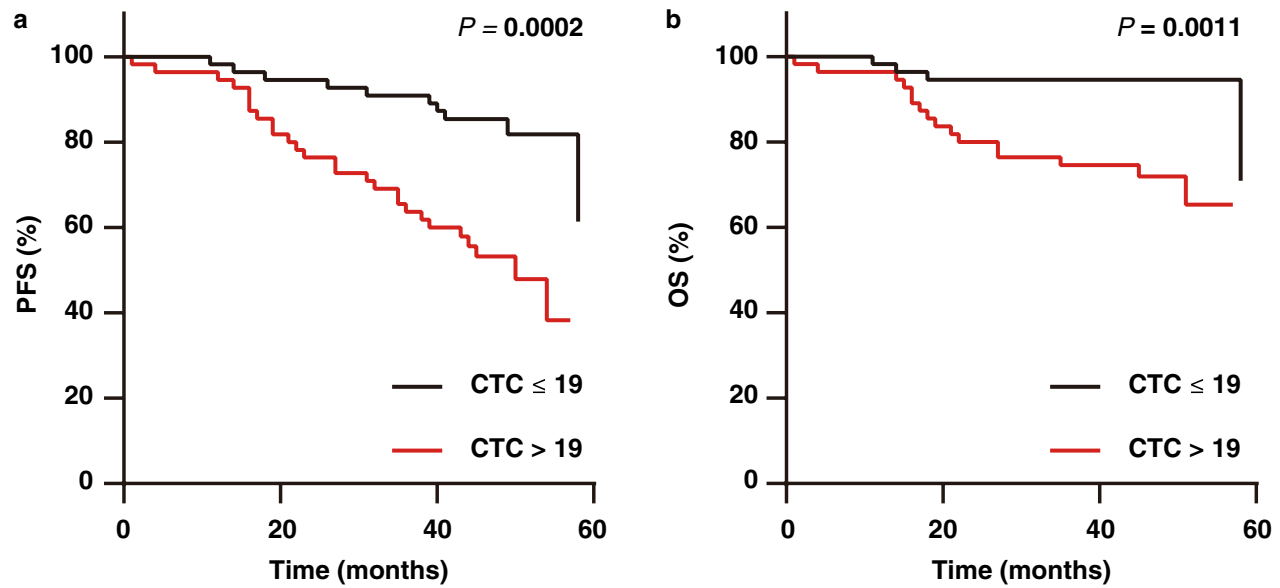

Figure 2. Kaplan-Meier curves showing estimated PFS (a) and OS (b) for patients with baseline CTC negativity $(\mathrm{CTC} \leq 19)$ and CTC positivity $(\mathrm{CTC}>19)$.

\begin{tabular}{|c|c|c|c|c|c|c|c|c|}
\hline & \multicolumn{4}{|l|}{ PFS } & \multicolumn{4}{|l|}{ OS } \\
\hline & \multicolumn{2}{|c|}{ Cox univariate analysis } & \multicolumn{2}{|c|}{ Cox multivariate analysis } & \multicolumn{2}{|c|}{ Cox univariate analysis } & \multicolumn{2}{|c|}{ Cox multivariate analysis } \\
\hline & HR (95\% CI) & $P$ value & HR $(95 \%$ CI $)$ & $P$ value & HR (95\% CI) & $P$ value & HR (95\% CI) & $P$ value \\
\hline CTC at baseline & $3.93(1.84-8.39)$ & 0.000 & $3.63(1.60-8.27)$ & 0.002 & $6.50(1.89-22.36)$ & 0.003 & $4.35(1.05-17.98)$ & 0.042 \\
\hline Intrinsic subtype & $1.19(0.91-1.55)$ & 0.197 & $1.00(0.61-1.66)$ & 0.986 & $1.24(0.87-1.77)$ & 0.235 & $0.92(0.40-2.14)$ & 0.855 \\
\hline Stage & $2.17(1.57-3.00)$ & 0.000 & $1.75(1.05-2.90)$ & 0.031 & $4.22(2.32-7.68)$ & 0.000 & $3.79(1.57-9.14)$ & 0.003 \\
\hline Risk categories & $3.68(1.94-7.00)$ & 0.000 & $1.66(0.62-4.44)$ & 0.309 & $8.99(3.04-26.57)$ & 0.000 & $2.07(0.48-8.96)$ & 0.333 \\
\hline Histology & $1.59(0.76-3.33)$ & 0.218 & $1.81(0.72-4.57)$ & 0.210 & $1.69(0.63-4.52)$ & 0.299 & $3.27(0.64-16.81)$ & 0.156 \\
\hline ER status & $0.52(0.27-1.01)$ & 0.054 & $0.43(0.14-1.32)$ & 0.139 & $0.41(0.17-1.00)$ & 0.049 & $0.17(0.02-1.33)$ & 0.091 \\
\hline PR status & $0.40(0.19-0.86)$ & 0.019 & $0.47(0.20-1.12)$ & 0.089 & $0.46(0.17-1.28)$ & 0.136 & $0.53(0.14-1.97)$ & 0.343 \\
\hline HER2 status & $1.07(0.55-2.08)$ & 0.833 & $0.78(0.34-1.79)$ & 0.561 & $1.06(0.44-2.57)$ & 0.900 & $0.37(0.10-1.41)$ & 0.146 \\
\hline Ki67 status & $1.97(0.90-4.33)$ & 0.091 & $1.16(0.46-2.92)$ & 0.760 & $3.06(0.90-10.46)$ & 0.074 & $2.02(0.36-11.41)$ & 0.426 \\
\hline Final model & & & & 0.000 & & & & 0.000 \\
\hline
\end{tabular}

Table 2. Cox univariate analysis and multivariate analysis of CTCs at baseline.

Prognostic relevance of CTC at the end-point follow-up visit. To investigate whether CTCs during the later phase of disease treatment are associated with prognosis, the prognostic relevance of CTCs at the end-point follow-up visit in 86 patients was analysed. There were 39 CTC-positive cases (45.3\%) and 47 CTCnegative cases $(54.7 \%)$. Twenty CTC-positive patients $(51.3 \%)$ developed disease recurrence, while disease recurrence occurred in only 7 CTC-negative patients (14.9\%). Twelve CTC-positive patients (30.8\%) died, while death only occurred in 3 CTC-negative patients (6.4\%). CTC-positive cases showed a significantly shorter PFS $(P=0.0003$; HR, $4.25 ; 95 \% \mathrm{CI}, 1.96-9.09)$ and $\mathrm{OS}(P=0.0028 ; \mathrm{HR}, 5.52 ; 95 \% \mathrm{CI}, 1.98-15.37)$ than CTC-negative cases (Fig. $5 \mathrm{a}$ and b). Therefore, CTCs at the end-point follow-up visit were associated with prognosis.

In univariable cox regression analysis, CTCs at the end-point-follow-up visit $(P=0.001 ; \mathrm{HR}, 4.21 ; 95 \% \mathrm{CI}$, $1.77-9.99)$, stage $(P=0.000 ; \mathrm{HR}, 2.55 ; 95 \% \mathrm{CI}, 1.70-3.83)$, risk categories $(P=0.000 ; \mathrm{HR}, 4.65 ; 95 \% \mathrm{CI} 2.12-10.19)$ and PR status ( $P=0.007 ; \mathrm{HR}, 0.26 ; 95 \%$ CI $0.10-0.69)$ were the significate factor of PFS (Table 3). In Cox multivariate analysis, CTCs at the end-point-follow-up visit $(P=0.010$; HR, 3.51; 95\% CI, 1.35-9.13) and PR status $(P=0.023 ; \mathrm{HR} 0.27 ; 95 \%$ CI, 0.09-0.84) were significantly correlated (Table 3), indicating that CTCs at the end-point-follow-up visit was the independent factor for PFS. For OS, CTCs at the end-point-follow-up visit $(P=0.009 ; \mathrm{HR}, 5.39 ; 95 \% \mathrm{CI}, 1.52-19.11)$, stage $(P=0.000 ; \mathrm{HR}, 5.22 ; 95 \% \mathrm{CI}, 2.53-10.78)$ and risk categories $(P=0.001 ; \mathrm{HR}, 28.78 ; 95 \%$ CI 3.81-217.68) were related to unfavourable prognosis (Table 3). However, CTCs at the end-point-follow-up visit wasn't the independent factor for OS in multivariable models with clinical covariates (Table 3 ).

\section{Discussion}

CTCs have been proven associated with the prognosis of breast cancer. However, therapy can affect CTCs, especially surgery ${ }^{15}$. It has been reported that the number of CTCs increases 3-4 days after surgery ${ }^{17}$. Our data showed that the number and prevalence of CTCs increased at $1 \mathrm{~d}$ and $7 \mathrm{~d}$ after surgery. No significant differences in PFS and OS were observed between days 1 and 7 after surgery for CTC-positive and CTC-negative patients, but CTCs measured on day 14 after surgery were related to PFS. Therefore, the measurement time point of CTCs affects their prognostic value. 

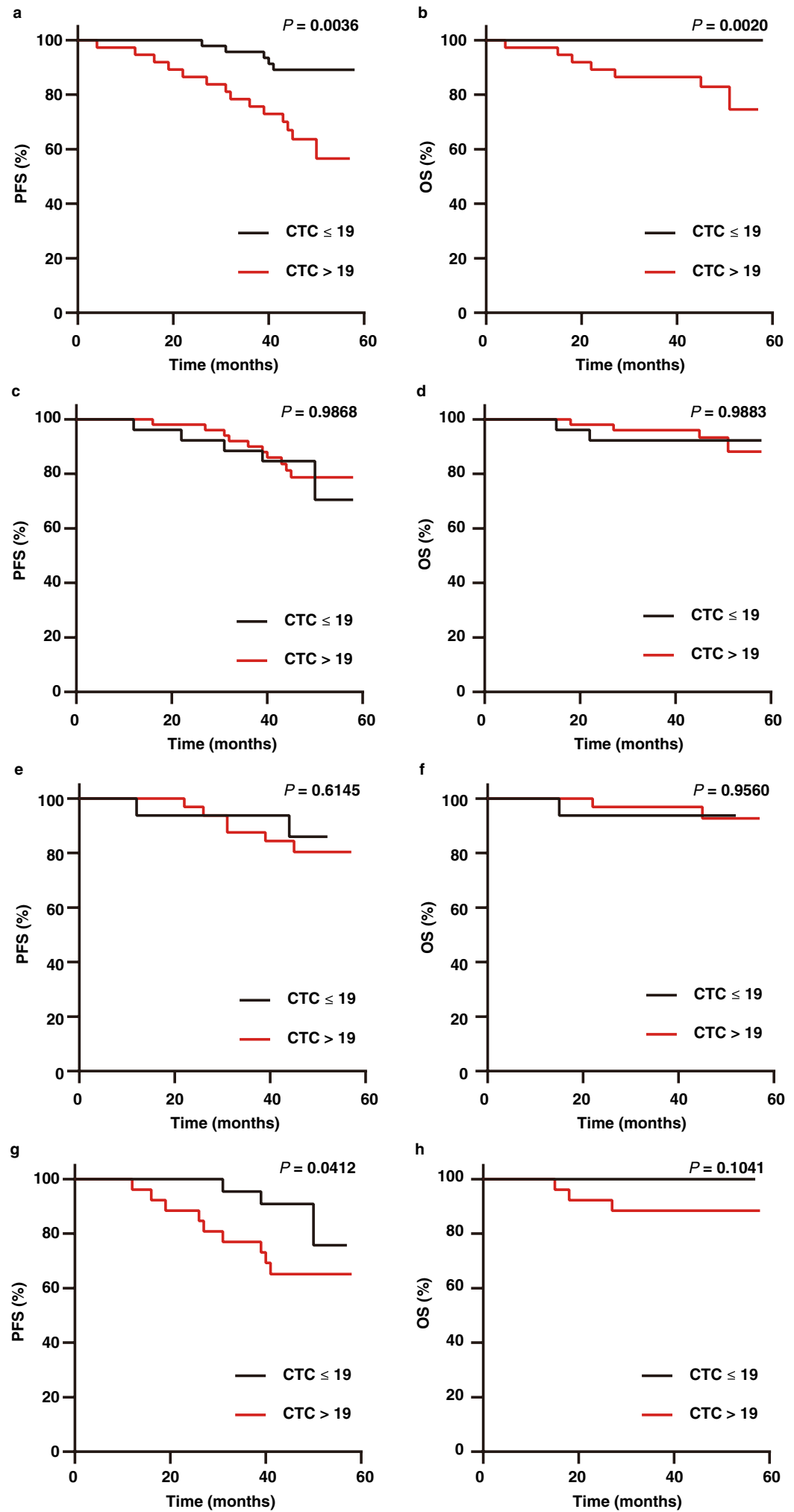

h

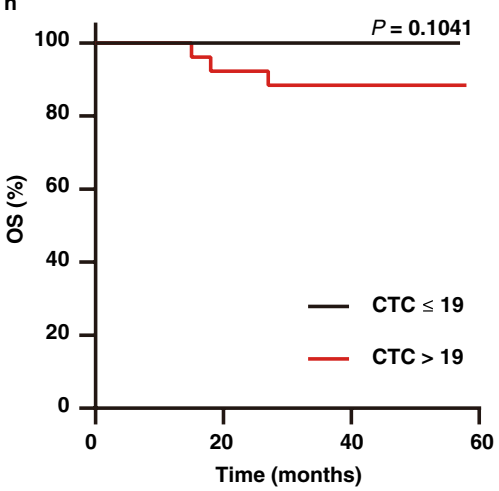

Figure 3. Kaplan-Meier curves showing estimated PFS and OS for patients with CTC negativity (CTC $\leq 19$ ) and CTC positivity $(\mathrm{CTC}>19)$ before surgery $(\mathbf{a}, \mathbf{b}), 1 \mathrm{~d}$ after surgery $(\mathbf{c}, \mathbf{d}), 7 \mathrm{~d}$ after surgery $(\mathbf{e}, \mathbf{f})$ and $14 \mathrm{~d}$ after surgery $(\mathbf{g}, \mathbf{h})$.

CTCs at baseline showed prognostic relevance in many reports ${ }^{26-28}$ as well as in the present study, because most CTCs at baseline were measured before any new line of treatment thus the effects of therapy were limited. 

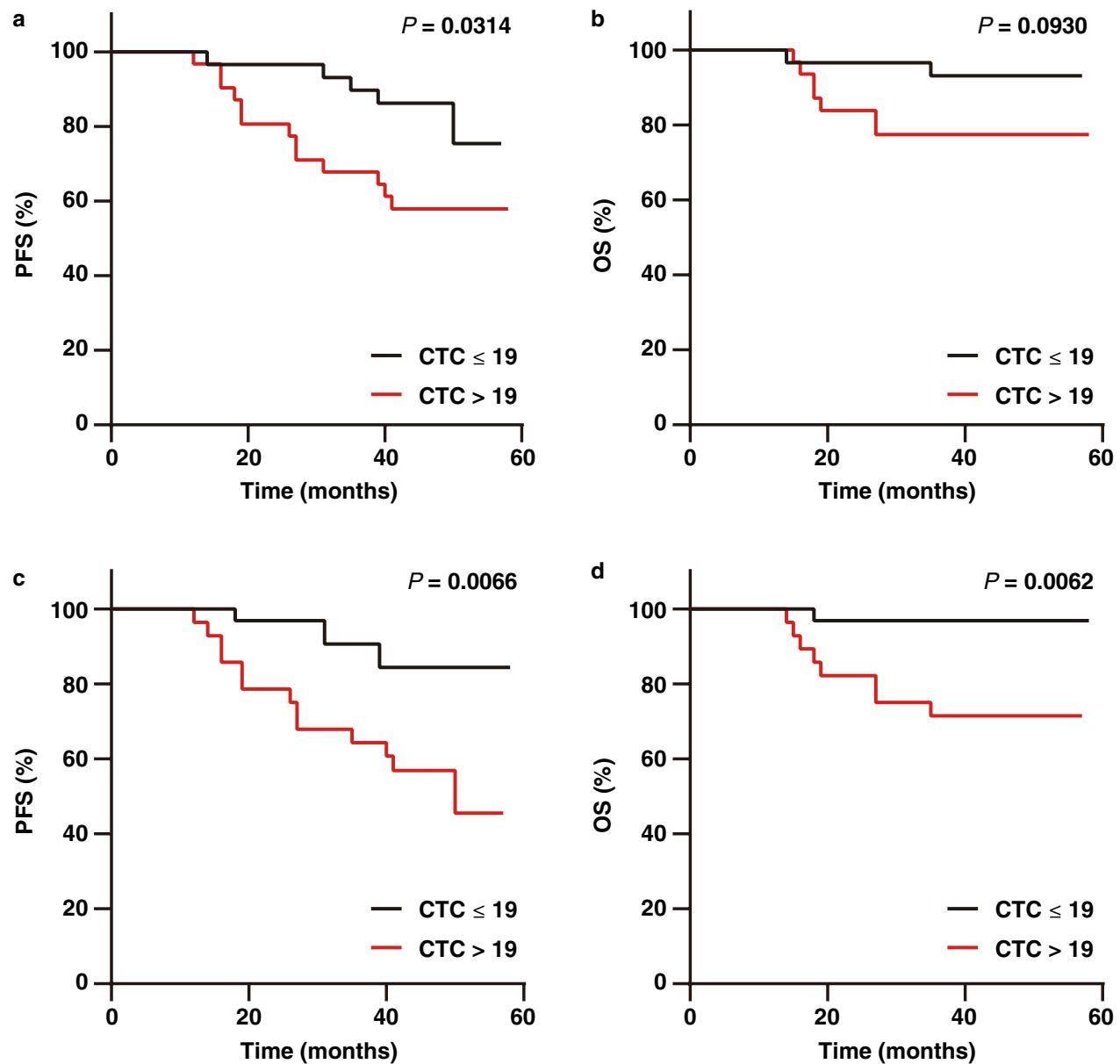

Figure 4. Kaplan-Meier curves showing estimated PFS and OS for patients with CTC negativity $(\mathrm{CTC} \leq 19)$ and CTC positivity (CTC > 19) before initial chemotherapy $(\mathbf{a}, \mathbf{b})$ and before final chemotherapy $(\mathbf{c}, \mathbf{d})$.
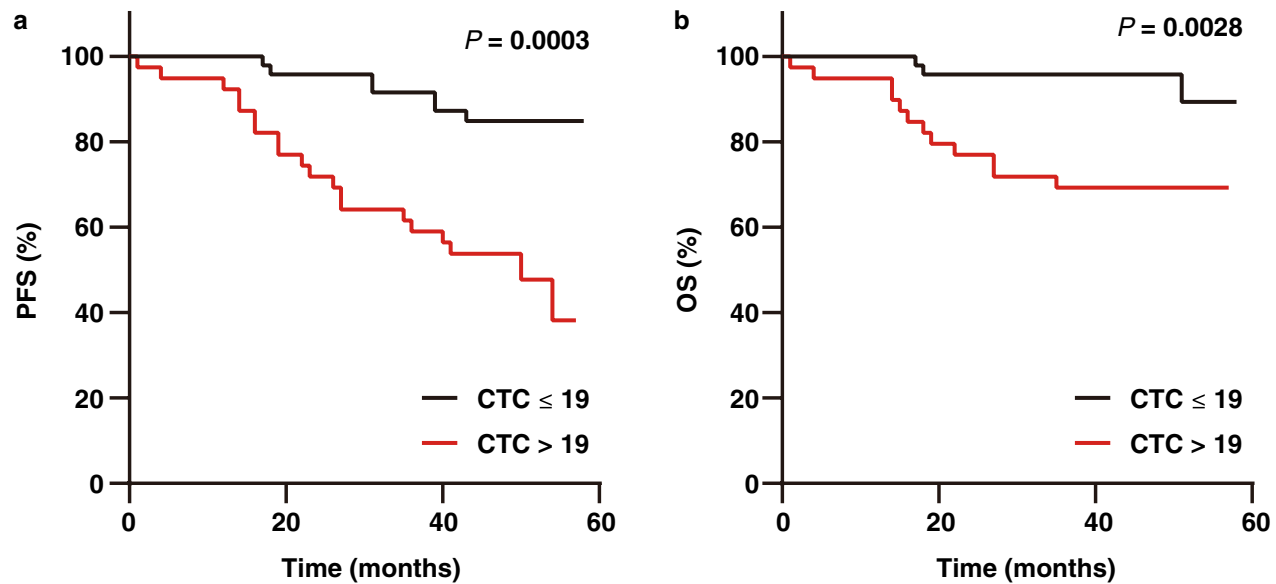

Figure 5. Kaplan-Meier curves showing estimated PFS (a) and OS (b) for patients with CTC positivity $(\mathrm{CTC}>19)$ and CTC negativity $(\mathrm{CTC} \leq 19)$ at the end-point follow-up visit.

However, some patients missed the CTC measurement time point at baseline, therefore if these patients want to benefit from the prognosis of CTCs, another CTC-measurement time point, at which the number of CTCs has prognostic value, should be determined.

Therefore, we investigated the prognostic relevance of CTCs before and after adjuvant chemotherapy in addition to the perioperative period. The prevalence of CTCs before adjuvant chemotherapy (51.7\%) was higher 


\begin{tabular}{|c|c|c|c|c|c|c|c|c|}
\hline & \multicolumn{4}{|l|}{ PFS } & \multicolumn{4}{|l|}{ os } \\
\hline & \multicolumn{2}{|c|}{ Cox univariate analysis } & \multicolumn{2}{|c|}{ Cox multivariate analysis } & \multicolumn{2}{|c|}{ Cox univariate analysis } & \multicolumn{2}{|c|}{ Cox multivariate analysis } \\
\hline & HR $(95 \%$ CI) & $P$ value & HR $(95 \%$ CI $)$ & $P$ value & HR $(95 \%$ CI $)$ & $P$ value & HR $(95 \% \mathrm{CI})$ & $P$ value \\
\hline $\begin{array}{l}\text { CTC at the end- } \\
\text { point follow-up } \\
\text { visit }\end{array}$ & $4.21(1.77-9.99)$ & 0.001 & $3.51(1.35-9.13)$ & 0.010 & $5.39(1.52-19.11)$ & 0.009 & $3.02(0.70-13.05)$ & 0.138 \\
\hline Intrinsic subtype & $1.30(0.97-1.74)$ & 0.084 & $0.82(0.44-1.51)$ & 0.517 & $1.30(0.88-1.93)$ & 0.193 & $0.72(0.26-1.94)$ & 0.511 \\
\hline Stage & $2.55(1.70-3.83)$ & 0.000 & $1.52(0.63-3.68)$ & 0.354 & $5.22(2.53-10.78)$ & 0.000 & $3.38(0.68-16.73)$ & 0.135 \\
\hline Risk categories & $4.65(2.12-10.19)$ & 0.000 & $2.40(0.49-11.60)$ & 0.278 & $\begin{array}{l}28.78(3.80- \\
217.68)\end{array}$ & 0.001 & \begin{tabular}{|l|}
$7.03(0.37-$ \\
$133.32)$
\end{tabular} & 0.194 \\
\hline Histology & $1.25(0.52-3.00)$ & 0.615 & $2.09(0.69-6.31)$ & 0.191 & $2.00(0.66-5.99)$ & 0.218 & $\begin{array}{l}10.48(1.72- \\
63.67)\end{array}$ & 0.011 \\
\hline ER status & $0.50(0.23-1.10)$ & 0.086 & $0.40(0.08-1.86)$ & 0.240 & $0.46(0.16-1.26)$ & 0.130 & $0.13(0.01-1.51)$ & 0.104 \\
\hline PR status & $0.26(0.10-0.69)$ & 0.007 & $0.27(0.09-0.84)$ & 0.023 & $0.48(0.15-1.51)$ & 0.208 & $0.41(0.08-2.09)$ & 0.285 \\
\hline HER2 status & $1.12(0.52-2.39)$ & 0.777 & $0.69(0.26-1.80)$ & 0.444 & $1.16(0.42-3.20)$ & 0.773 & $0.50(0.10-2.51)$ & 0.399 \\
\hline Ki67 status & $2.06(0.83-5.11)$ & 0.120 & $1.24(0.40-3.82)$ & 0.709 & $3.71(0.84-16.50)$ & 0.085 & $1.09(0.18-6.64)$ & 0.926 \\
\hline Final model & & & & 0.000 & & & & 0.000 \\
\hline
\end{tabular}

Table 3. Cox univariate analysis and multivariate analysis of CTCs at the end-point follow-up visit.

than that after adjuvant chemotherapy (46.7\%), indicating that chemotherapy had an impact on CTCs. However, CTCs after chemotherapy showed more associations with PFS and OS than those before adjuvant chemotherapy, revealing that a CTC-measurement time point closer to the later phase of the treatment had a more obvious prognostic value. To validate this hypothesis, the prognostic relevance of CTCs at the end-point follow-up visit was investigated. Although the end-point follow-up visit did not occur after a fixed period, CTCs at the end-point follow-up visit showed a prognostic value. Therefore, in addition to the baseline, CTCs at the late phase of the disease or at the end of treatment also have prognostic value.

The research of the present study has some limitations: (1) It was a single-centre prospective study and the number of patients enrolled was limited. Patient stratification analysis did not proceed because of the limited number of patients. The mobility of patients resulted in a smaller sample size at follow-up visit and the treatment monitoring; (2) Although the performance of IMNs for CTC collection was validated, this method was not compared to other platforms such as CellSearch system; (3) More treatment stages, such as neoadjuvant chemotherapy, should be considered for inclusion in a study to assess the prognostic value of CTCs measured at different time points.

\section{Conclusion}

In this prospective trial, the prognostic value of the timing of CTC measurement for breast cancer in the Chinese population was demonstrated. CTCs change dynamically during treatment, which can affect prognosis. Surgery led to an increase in the number and prevalence of CTCs on the first day after surgery and did not return to the preoperative level until $14 \mathrm{~d}$ after surgery. The CTC prevalence at the baseline and end-point follow-up visits was related to PFS and OS, while the CTCs detected before chemotherapy were only related to PFS. The CTCs detected at the last adjuvant chemotherapy were more correlated with prognosis than those before adjuvant chemotherapy in the analysis of paired samples. Therefore, CTCs at baseline or in the late phase of treatment are preferable for prognosis.

\section{Materials and methods}

Patients and study design. Between 2015 and 2016, 164 patients with breast disease who were diagnosed or treated at Jinling Hospital were included in our study. Peripheral blood was collected before surgery and recorded as a baseline then when available before each treatment until the end of the adjuvant chemotherapy. A discard tube was used to collect the first $1 \mathrm{~mL}$ of bloods to avoid epithelial skin cells contamination. Progression or non-progression was evaluated according to clinical practice based on clinical and radiological evaluations. Peripheral blood specimens from 19 healthy donors were used as negative controls.

The study was approved by the Committee on Biomedical Research Ethics of Jinling Hospital (2015NZGKJ082) and was performed in accordance with the Declaration of Helsinki. All participants provided written informed consent.

Cell culture. MCF-7 (HTB-22., ATCC, VA, USA), HCT-116 (CCL-247., ATCC), and A549 (CCL-158., ATCC) were grown in a $25-\mathrm{cm}^{2}$ flask containing high-glucose Dulbecco's modified Eagle's medium (DMEM) (Gibco, NY, USA) supplemented with $10 \%$ fetal bovine serum (FBS) (Gibco) under $5 \% \mathrm{CO}_{2}$ at $37{ }^{\circ} \mathrm{C}$.

Capture of spiked tumour cells in mock samples. MCF-7 cells (passage14) were placed in phosphatebuffered saline (PBS) as mock samples. CTCs were captured by quick-response immunomagnetic nanosphere (IMN)-modified anti-epithelial-cell-adhesion-molecule (EpCAM) monoclonal antibody (Wuhan Jiayuan Quantum Dots Corporation, Ltd., Wuhan, China $)^{21}$. IMNs were added to $7.5 \mathrm{~mL}$ of sample, and the mixture 
was incubated for $5 \mathrm{~min}$ at $37^{\circ} \mathrm{C}$. After magnetic separation, the captured cells were fixed with $4 \%$ paraformaldehyde (Nanjing Chemical Reagent Co., Ltd., Nanjing, China), permeabilized, blocked with 0.1\% Triton-X 100 (Sigma-Aldrich, MO, USA) and 1\% bovine serum albumin (BSA) (Amresco, OH, USA), and stained with $30 \mu \mathrm{g} /$ mL 4',6-diamidino-2-phenylindole (DAPI) (Sigma-Aldrich), FITC-labeled anti-keratin-19 (CK19) monoclonal antibody (Abcam, MA, USA), and APC-labelled anti-protein-tyrosine-phosphatase-receptor-type-C (CD45) monoclonal antibody (Abcam). After washing, the captured cells were placed in a cell culture dish (Greiner, Kremsmünster, Austria) for fluorescence microscopy imaging. CTCs were identified as cells that had round to oval morphology, were positive for DAPI and CK19, and negative for CD45. All processes are completed within $2 \mathrm{~h}$.

Detection of CTCs in breast disease patients and healthy donors. Eight millilitres of blood was drawn into EDTAVacutainer tubes (BD, NJ, USA). The blood samples were stored at $4{ }^{\circ} \mathrm{C}$ and processed within two $\mathrm{h}$ after collection. Seven point five millilitres of blood was incubated with IMNs for 5 min. After enrichment, the subsequent process is the same as the process described in "Capture of spiked tumour cells in mock samples" section.

Statistical analysis. Statistical analyses were performed using IBM SPSS Statistics (version 24.0, IBM, Armonk, NY, USA). Pearson's chi-square test was used to compare the categorical variables. Pearson's chisquared test for trend was used to compare the ordinal data. The Mann-Whitney U test was used to compare continuous variables. Kaplan-Meier estimator and log-rank tests were used to compare survival rates between groups. Cox proportional hazards regression was used to determine the univariate and multivariate hazard ratios (HRs) of the potential predictors of PFS and OS.

Received: 17 September 2020; Accepted: 16 June 2021

Published online: 29 June 2021

\section{References}

1. Gkountela, S. et al. Circulating tumor cell clustering shapes DNA methylation to enable metastasis seeding. Cell 176, 98-112. https://doi.org/10.1016/j.cell.2018.11.046 (2019).

2. Massagué, J. \& Obenauf, A. C. Metastatic colonization by circulating tumour cells. Nature 529, 298-306. https://doi.org/10.1038/ nature17038 (2016).

3. Dasgupta, A., Lim, A. R. \& Ghajar, C. M. Circulating and disseminated tumor cells: harbingers or initiators of metastasis?. Mol. Oncol. 11, 40-61. https://doi.org/10.1002/1878-0261.12022 (2017).

4. Pantel, K. \& Speicher, M. R. The biology of circulating tumor cells. Oncogene 35, 1216-1224. https://doi.org/10.1038/onc.2015. $192(2016)$.

5. Bidard, F. C., Proudhon, C. \& Pierga, J. Y. Circulating tumor cells in breast cancer. Mol. Oncol. 10, 418-430. https://doi.org/10. 1016/j.molonc.2016.01.001 (2016).

6. Kalinich, M. et al. An RNA-based signature enables high specificity detection of circulating tumor cells in hepatocellular carcinoma. Proc. Natl. Acad. Sci. U. S. A. 114, 1123-1128. https://doi.org/10.1073/pnas.1617032114 (2017).

7. Cristofanilli, M. et al. Circulating tumor cells, disease progression, and survival in metastatic breast cancer. N. Engl. J. Med. 351, 781-791. https://doi.org/10.1056/NEJMoa040766 (2004).

8. Smerage, J. B. et al. Circulating tumor cells and response to chemotherapy in metastatic breast cancer: SWOG S0500. J. Clin. Oncol. 32, 3483-3489. https://doi.org/10.1200/jco.2014.56.2561 (2014).

9. Carter, L. et al. Molecular analysis of circulating tumor cells identifies distinct copy-number profiles in patients with chemosensitive and chemorefractory small-cell lung cancer. Nat. Med. 23, 114-119. https://doi.org/10.1038/nm.4239 (2017).

10. Bidard, F. C. et al. Clinical validity of circulating tumour cells in patients with metastatic breast cancer: a pooled analysis of individual patient data. Lancet Oncol. 15, 406-414. https://doi.org/10.1016/s1470-2045(14)70069-5 (2014).

11. Pierga, J. Y. et al. High independent prognostic and predictive value of circulating tumor cells compared with serum tumor markers in a large prospective trial in first-line chemotherapy for metastatic breast cancer patients. Ann. Oncol. 23, 618-624. https://doi. org/10.1093/annonc/mdr263 (2012).

12. Hayes, D. F. et al. Circulating tumor cells at each follow-up time point during therapy of metastatic breast cancer patients predict progression-free and overall survival. Clin. Cancer Res. 12, 4218-4224. https://doi.org/10.1158/1078-0432.ccr-05-2821 (2006).

13. Edge, S. B. \& Compton, C. C. The American Joint Committee on Cancer: the 7th edition of the AJCC cancer staging manual and the future of TNM. Ann. Surg. Oncol. 17, 1471-1474. https://doi.org/10.1245/s10434-010-0985-4 (2010).

14. Rack, B. et al. Circulating tumor cells predict survival in early average-to-high risk breast cancer patients. J. Natl. Cancer Inst. 106, dju066. https://doi.org/10.1093/jnci/dju066 (2014).

15. Hall, C. S. et al. Prognostic value of circulating tumor cells identified before surgical resection in nonmetastatic breast cancer patients. J. Am. Coll. Surg. 223, 20-29. https://doi.org/10.1016/j.jamcollsurg.2016.02.021 (2016).

16. Ramirez, J. M. et al. Prognostic relevance of viable circulating tumor cells detected by EPISPOT in metastatic breast cancer patients. Clin. Chem. 60, 214-221. https://doi.org/10.1373/clinchem.2013.215079 (2014).

17. Camara, O. et al. Seeding of epithelial cells into circulation during surgery for breast cancer: the fate of malignant and benign mobilized cells. World J. Surg. Oncol. 4, 67. https://doi.org/10.1186/1477-7819-4-67 (2006).

18. van Dalum, G. et al. Circulating tumor cells before and during follow-up after breast cancer surgery. Int. J. Oncol. 46, 407-413. https://doi.org/10.3892/ijo.2014.2694 (2015).

19. Paoletti, C. et al. Significance of circulating tumor cells in metastatic triple-negative breast cancer patients within a randomized, phase II Trial: TBCRC 019. Clin. Cancer. Res. 21, 2771-2779. https://doi.org/10.1158/1078-0432.ccr-14-2781 (2015).

20. Smerage, J. B. et al. Monitoring apoptosis and Bcl-2 on circulating tumor cells in patients with metastatic breast cancer. Mol. Oncol. 7, 680-692. https://doi.org/10.1016/j.molonc.2013.02.013 (2013).

21. Congying, W. et al. Quick-response magnetic nanospheres for rapid, efficient capture and sensitive detection of circulating tumor cells. ACS Nano 8, 941-949 (2014).

22. Aurilio, G. et al. Prognostic value of circulating tumor cells in primary and metastatic breast cancer. Expert Rev. Anticancer Ther. 12, 203-214. https://doi.org/10.1586/era.11.208 (2012). 
23. Xenidis, N. et al. Cytokeratin-19 mRNA-positive circulating tumor cells after adjuvant chemotherapy in patients with early breast cancer. J. Clin. Oncol. 27, 2177-2184. https://doi.org/10.1200/jco.2008.18.0497 (2009).

24. Cristofanilli, M. et al. Circulating tumor cells: a novel prognostic factor for newly diagnosed metastatic breast cancer. J. Clin. Oncol. 23, 1420-1430. https://doi.org/10.1200/jco.2005.08.140 (2005).

25. Helissey, C. et al. Circulating tumor cell thresholds and survival scores in advanced metastatic breast cancer: the observational step of the CirCe01 phase III trial. Cancer Lett. 360, 213-218. https://doi.org/10.1016/j.canlet.2015.02.010 (2015).

26. Tokudome, N. et al. Detection of circulating tumor cells in peripheral blood of heavily treated metastatic breast cancer patients. Breast Cancer 18, 195-202. https://doi.org/10.1007/s12282-011-0259-4 (2011)

27. Larsson, A. M. et al. Longitudinal enumeration and cluster evaluation of circulating tumor cells improve prognostication for patients with newly diagnosed metastatic breast cancer in a prospective observational trial. Breast Cancer Res. 20, 48. https://doi. org/10.1186/s13058-018-0976-0 (2018).

28. Franken, B. et al. Circulating tumor cells, disease recurrence and survival in newly diagnosed breast cancer. Breast Cancer Res. 14, R133. https://doi.org/10.1186/bcr3333 (2012).

\section{Acknowledgements}

This work was supported by the State Key Basic Research Program of the PRC (2016YFA0201204, 2014CB744501), National Natural Science Foundation of China (61871403), Jiangsu Provincial Science Fund for Distinguished Young Scholars (BK20180005), Jiangsu Provincial Medical Youth Talent Program (No. QNRC2016889).

\section{Author contributions}

S.Y.P., B.J.Z., S.H.W., and G.H.Z. wrote and reviewed the manuscript. S.Y.P., H.J.L., S.X., B.J.Z., S.H.W., and G.H.Z. contributed to the experimental design. S.Y.P., H.J.L., S.X., L.Y.F., X.P.M., and Y.N.C. performed the experiments. H.J.L. and S.H.W. collected the patients' peripheral blood and gathered the patients' clinical data. All authors contributed to data analysis and interpretation, and read and approved of the final manuscript.

\section{Competing interests}

The authors declare no competing interests.

\section{Additional information}

Supplementary Information The online version contains supplementary material available at https://doi.org/ 10.1038/s41598-021-92876-8.

Correspondence and requests for materials should be addressed to B.Z., S.W. or G.Z.

Reprints and permissions information is available at www.nature.com/reprints.

Publisher's note Springer Nature remains neutral with regard to jurisdictional claims in published maps and institutional affiliations.

(c) Open Access This article is licensed under a Creative Commons Attribution 4.0 International License, which permits use, sharing, adaptation, distribution and reproduction in any medium or format, as long as you give appropriate credit to the original author(s) and the source, provide a link to the Creative Commons licence, and indicate if changes were made. The images or other third party material in this article are included in the article's Creative Commons licence, unless indicated otherwise in a credit line to the material. If material is not included in the article's Creative Commons licence and your intended use is not permitted by statutory regulation or exceeds the permitted use, you will need to obtain permission directly from the copyright holder. To view a copy of this licence, visit http://creativecommons.org/licenses/by/4.0/.

(c) The Author(s) 2021 